\title{
CD300LG improves the cytotoxic activity of CIK
}

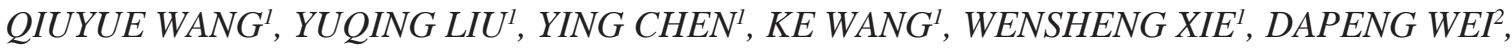 \\ LIJUAN HU \\ ${ }^{1}$ College of Preclinical and Forensic Medicine, Sichuan University, China \\ ${ }^{2}$ Department of Immunology, College of Preclinical and Forensic Medicine, Sichuan University, Chengdu, China
}

\begin{abstract}
To investigate the effect of CD300LG- $\gamma$ induction on the cytotoxic activity of CIK. Eukaryotic expression plasmid hCD300LG- $\gamma /$ pEGFP-C3, which can express human CD300LG- $\gamma$, was constructed and transfected into $\mathrm{CHO}$ cells by lipofectamine. The expression of CD30OLG- $\gamma$ was confirmed by immunofluorescence, RT-PCR, and Western Blot. To produce CIK cells, human peripheral blood mononuclear cells (PBMC) were isolated and induced, respectively, by cell lysates extracted from hCD300LG- $\gamma / C H O$ cells, pEGFP-C3/CHO cells, and CHO cells, concurrently with the standard CIK inductive agent. The

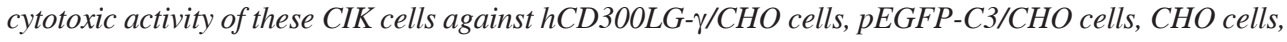
and K562 cells was tested. The results showed that eukaryotic expression of plasmid hCD300LG- $\gamma /$ pEGFP-C3 was constructed and transfected into CHO cells successfully. After being induced by cell lysates, the cytotoxicity of hCD300LG- $\gamma / C H O-C I K$ was improved compared with the other CIK cells. In particular, the activity of killing $\mathrm{pEGFP-C3/CHO}$ and $\mathrm{CHO}$ cells was improved significantly. Meanwhile, the activity of $h C D 300 L G-\gamma / C H O-C I K$ killing K562 was improved significantly compared with the other CIK cells. The results indicated that hCD300LG- $\gamma$ induction can significantly improve the killing activity of CIK cells.
\end{abstract}

Key words: CD300LG, cytokine induced killer (CIK), eukaryotic expression plasmid, transfect, killing activity.

(Cent Eur J Immunol 2017; 42 (2): 117-122)

CD300LG (CD300 antigen-like family member G), also called nepmucin or CLM-9, is a novel sialomucin belonging to type-I membrane protein, which contains a hydrophobic signal peptide, a single V-type Ig domain, a classical mucin-like domain, a transmembrane domain, and an intracellular domain [1]. CD300LG is expressed extensively in a variety of organisational venules and capillary endothelial cells, such as cardiac, liver, spleen, thymus, lung, kidney, skeletal muscle, salivary gland, thyroid, prostate, tongue, peripheral lymph nodes, and high endothelial vein (HEV) except Payer patch [2]. However, in immunological privileged sites, such as brain, testis, uterus, and gut, in which the immunotolerance is easy to induce, the expression of CD300LG is very low or absent [3].

CD300LG plays an important role in promoting lymphocyte transendothelial migration (TEM) of HEVs [1]. Appropriately glycosylated CD300LG with MECA-79 epitope can associate with L-selectin to mediate L-selectin-dependent lymphocyte rolling by its mucin-like domain [2]. In the adhesion step, CD300LG mediates lymphocyte adhesion with endothelial cells (ECs) by its Ig domain, which is independent of LFA-1 or VLA-4 adhesion path- way [1]. In addition, CD300LG promotes lymphocyte transmigration through ECs by its Ig domain $[4,5]$.

Many studies have demonstrated that adhesion molecules are correlated with tumorogenesis, development, and metastasis. Adhesion molecules participate in the interaction between tumour cells and anti-tumour effector cells, such as CTL, NK, macrophage, etc. [6, 7]. Various adhesion molecules, such as E-cadherin, $\mathrm{N}$-cadherin, and integrins, are closely related with tumour invasion and metastasis [8-12]. Consequently, adhesion molecules have become target molecules in biotherapy of tumours, autoimmune diseases, inflammatory disease, etc. [13, 14].

Umemoto E et al. found that the expression of CD300LG was down-regulated in tumours and tumour-draining lymph nodes, indicating that the expression of CD300LG was negatively regulated by locally produced signals under these circumstances [3], whereas in chronic pancreatic inflammation, CD300LG was up-regulated, and there were a large number of activated $\mathrm{CD} 4^{+} \mathrm{T}$ cells infiltrating the pancreas [3]. Our previous studies also demonstrated that both mRNA and protein expression levels of CD300LG- $\gamma$ in pulmonary carcinoma tissues were signifi-

Correspondence: Lijuan Hu, Department of Immunology, College of Preclinical and Forensic Medicine, Sichuan University, Chengdu, 610041, China, tel. 13982076246; fax 85501606; e-mail: hulijuan@scu.edu.cn Submitted: 1.01.2016; Accepted: 18.04.2016 
cantly decreased compared with that in tumour-adjacent tissues, which might lead to inhibition of killing activity of immunocytes, resulting in immune escape of lung cancer cells [15].

As discussed above, CD300LG has been indicated to be closely correlated with onset and development of tumours. As a novel adhesion molecule, it is bound to participate in and mediate the interactions of immunocytes and tumour cells. So, eukaryotic expression plasmid that can express hCD300LG- $\gamma$ was constructed and transfected into CHO cells to obtain hCD300LG- $\gamma / \mathrm{CHO}$. Using cell lysates as an inducer combined with standard CIK induction agent, four kinds of CIK were obtained. By comparing the activity of these CIK killing target cells, the effect of CD300LG induction on killing activities of CIK was preliminarily studied. The results may facilitate elucidation of antitumor activity of CD300LG and provide a theoretical basis for application of DC-CIK in tumour immunotherapy.

\section{Material and methods}

\section{Material}

Strains, plasmids, and cell lines: JM109, recombinant hCD300LG- $\gamma /$ Pet41a-c(+) plasmid, eukaryotic expression plasmid-pEGFP-C3, CHO cells, and K562 cells (provided by the Department of Immunology, College of Preclinical and Forensic Medicine, Sichuan University).

Main reagents: Plasmid Mini Kit (Omega); DNA Gel Extraction Min Kit (Omega); HindIII and EcoRI (Takara); primers (Invitrogen); Eukaryotic transfection reagent: Lipofectamine ${ }^{\mathrm{TM}} 2,000$ (Invitrogen); Antibodies: Anti GFP antibody and HRP-conjugated goat anti-mouse IgG (ZSJQ Biological Technology Company, Beijng); G418 (Amresco); Trizol (Invitrogen), One-step RT-PCR kit (TaKaRa); Standard CIK inducer (Invitrogen); AlamarBlue cytotoxicity assay kit (Keygen).

\section{Methods}

\section{Construction and identification of eukaryotic expression plasmid hCD300LG- $\gamma /$ pEGFP-C3}

Using recombinant hCD300LG- $\gamma / \operatorname{Pet} 41 \mathrm{a}-\mathrm{c}(+)$ plasmid as a template, human CD300LG- $\gamma$ gene was amplified by PCR using forward primer 5'-AACCAAGCTTCTGGAGGGCCCAGAGGAAATCAG-3' and reverse primer 5'-CCAAGAATTCGACCATCGGGATGGACACCCTG-3'. PCR amplification condition was $95^{\circ} \mathrm{C} 3 \mathrm{~min}$; $95^{\circ} \mathrm{C} 30 \mathrm{~s}, 56^{\circ} \mathrm{C} 30 \mathrm{~s}, 72^{\circ} \mathrm{C} 45 \mathrm{~s}\left(34\right.$ cycles); $72^{\circ} \mathrm{C} 5 \mathrm{~min}$. Amplified products were purified by DNA Gel Extraction Min Kit (Omega) and subcloned into pEGFP-C3 vector (Novagen, Merck KGaA, Darmstadt, Germany) between EcoRI and HindIII. Then the recombinant hCD300LG- $\gamma /$ pEGFP-C3 plasmid was transformed into E. coli JM109.
Positive clones with ampicillin resistance were selected and identified by colony PCR, dual enzymatic digestion, and gene sequencing.

\section{In vitro transfection of eukaryotic plasmid hCD300LG- $\gamma / p E G F P-C 3$ into CHO cells}

CHO cells were cultured with RPMI-1640 medium containing $10 \%$ foetal bovine serum at $37^{\circ} \mathrm{C}$ with $5 \% \mathrm{CO}_{2}$ in a humid incubator. According to the instruction of the eukaryotic transfection reagent Lipofectamine TM2,000 (LF2,000), hCD300LG- $\gamma /$ pEGFP-C3 plasmid was transfected into CHO cells and selected by G418 $(800 \mu \mathrm{g} / \mathrm{ml})$. Meanwhile, pEGFP-C3 plasmid transfected into $\mathrm{CHO}$ cells was used as negative control as well as $\mathrm{CHO}$ cells. After 60 days of selection, two stable transfected cells lines were obtained, which were named hCD300LG- $\gamma / \mathrm{CHO}$ cells and pEGFP-C3/CHO cells, respectively, and identified by immunofluorescence, RT-PCR, and Western Blot.

\section{Detection of the killing activities of induced CIK cells}

Preparation of cell lysates: hCD300LG- $\gamma / \mathrm{CHO}$ cells, PEGFP-C3/CHO cells, and CHO cells were cultured in RPMI-1640 medium containing 10\% serum. When cells were overgrown, the cells were digested by pancreatic enzyme, and centrifuged to collect cell precipitation. The precipitation was dissolved in $200 \mu \mathrm{l} 1 \times$ buffer, ultrasonically broken, and then boiled for 5 min to obtain cell lysates as inducing factors.

CIK induction: The peripheral blood mononuclear cells (PBMC) were separated from peripheral blood cells by Ficol and equally divided into four parts, then transferred into four culture flasks. Firstly, standard CIK inducer (IL-1, IL-2, IFN- $\gamma$, and CD3McAb) were added into the four flasks, which were, respectively, marked as A1, A2, $\mathrm{A} 3$, and A4. Then the three kinds of cell lysates obtained from hCD300LG- $\gamma / \mathrm{CHO}$ cells, pEGFP-C3/CHO cells, and $\mathrm{CHO}$ cells were added into A1, A2, and A3, respectively, whereas A4 induced only with standard CIK inducer was used as a control. Finally, all cells in A1, A2, A3, and A4 were cultured at $37^{\circ} \mathrm{C}$ in a $5 \% \mathrm{CO}_{2}$ incubator for seven, days. Four kinds of induced CIK cells were obtained which were, respectively, named hCD300LG- $\gamma / \mathrm{CHO}-\mathrm{CIK}$, pEGFP-C3/CHO-CIK, CHO-CIK, and CK-CIK.

Detection of the killing activities of induced CIK cells: Using hCD300LG- $\gamma / \mathrm{CHO}-\mathrm{CIK}$, pEGFP-C3/CHOCIK, CHO-CIK, and CK-CIK as effector cells, and concurrently using hCD300LG- $\gamma / \mathrm{CHO}$ cells, pEGFP-C3/ $\mathrm{CHO}$ cells, $\mathrm{CHO}$ cells, and $\mathrm{K} 562$ cells as target cells, the cross-killing tests were carried out with a $10^{2}: 1$ ratio of effector cells to target cells on 96-well plates. The killing test wells, target control wells, and effector control wells were set. In the killing test wells, four kinds of target cells were killed by the four kinds of induced CIK cells. In tar- 

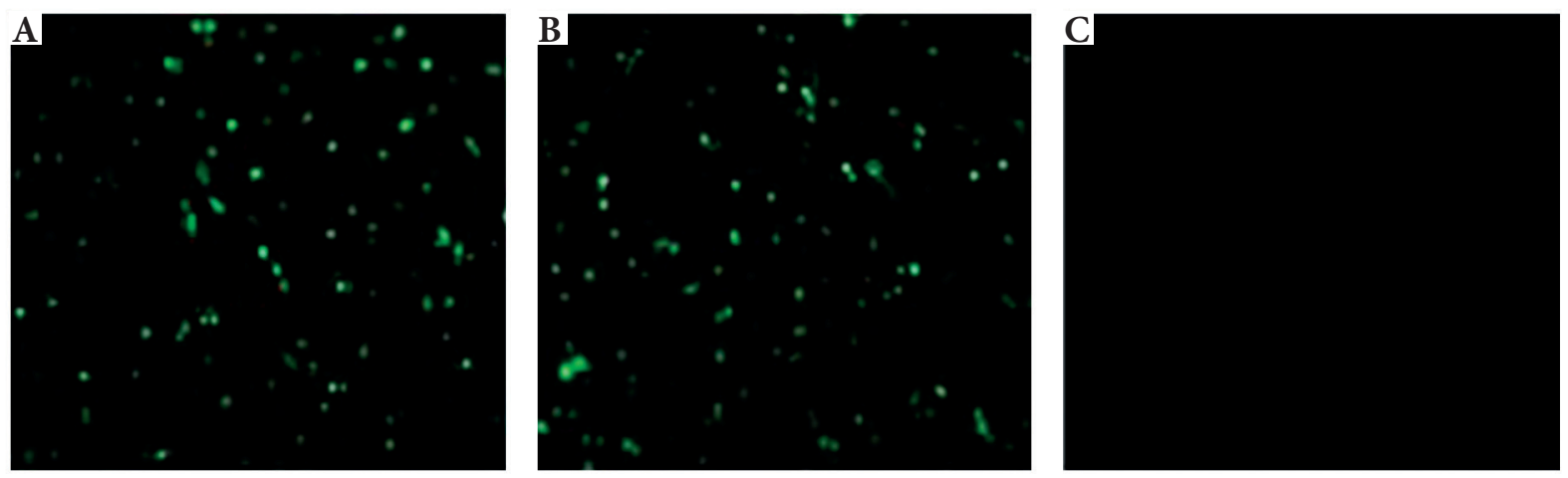

Fig. 1. Fluorescence microscope analysis of GFP tag expression in transfected CHO cells (100x). A) hCD300LG- $\gamma / \mathrm{CHO}$ cells; B) pEGFP-C3/CHO cells; C) $\mathrm{CHO}$ cells as negative control

get control wells, four kinds of target cells were the controls. In effector control wells, four kinds of effector cells were the controls. After 18 hours culture of effector cells mixed with target cells, AlamarBlue reagent was added into the wells $(10 \mu \mathrm{l}$ per well) and cultured in darkness for six hours. The light absorption value (A value) at $590 \mathrm{~nm}$ was recorded by colorimetric method. Each well was set triplicate wells, and the mean of triplicate wells was recorded, respectively, as $\mathrm{A}_{\text {test well, }}, \mathrm{A}_{\text {effector control well }}$, and $\mathrm{A}_{\text {target con- }}$ trol wells The killing activities of the induced CIK cells were calculated according to the following formula: Killing ratio $=\left[1-\left(\mathrm{A}_{\text {test well }}-\mathrm{A}_{\text {effector control well }}\right) / \mathrm{A}_{\text {target control wells }}\right] \times 100 \%$.

\section{Results}

\section{Construction and identification of eukaryotic expression plasmid hCD300LG- $\gamma /$ pEGFP-C3}

After hCD300LG- $\gamma /$ pEGFP-C3 plasmid transfection into JM109, positive clones with ampicillin-resistance were screened by colony PCR and identified by HindIII and EcoRI digestion (data not shown). Then the identified positive clone was confirmed by gene sequencing. The result showed that hCD300LG- $\gamma / \mathrm{pEGFP}-\mathrm{C} 3$ plasmid contained complete and correct human CD300LG- $\gamma$ gene without gene or frameshift mutation (data not shown).

\section{In vitro transfection of hCD300LG- $\gamma / p E G F P-C 3$ into $\mathrm{CHO}$ cells}

Cell immunofluorescence: The plasmids of hCD300LG- $\gamma /$ pEGFP-C3 and pEGFP-C3 were transfected into $\mathrm{CHO}$ cells. Then, the results were observed by fluorescence microscope to identify the expression of GFP tag (Fig. 1). There was no green fluorescence in the negative control, whereas hCD300LG- $\gamma / \mathrm{CHO}$ and pEGFP-C3/CHO cells both expressed obvious green fluorescent protein.

RT-PCR: The total RNA was extracted by Trizol. Then, genes were amplified by one-step RT-PCR with

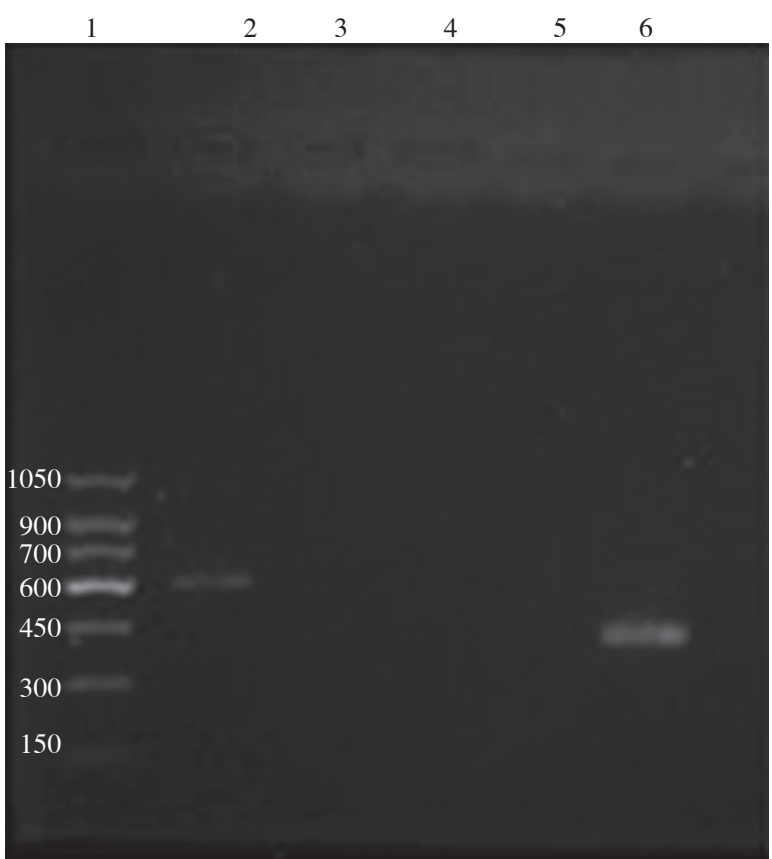

Fig. 2. RT-PCR analysis of human CD300LG- $\gamma$ gene expression: 1 - DNA maker; 2 - hCD300LG- $\gamma / \mathrm{CHO}$ cells; 3 - pEGFP-C $3 / \mathrm{CHO}$ cells; 4 - CHO cells; 5 - negative control; 6 - positive control

primers of 5'-CTGCTCGCTTCGCTACTTGGA-3 and 5'-CGGCACCTGTCCTACGAGTTG-3. RNA provided by the kit was used as a positive control, without adding a template as a negative control. As shown in Fig. 2, there were products in hCD300LG- $\gamma / \mathrm{CHO}$ cells (about $600 \mathrm{bp}$ ) and in positive control (about $450 \mathrm{bp}$ ), whereas there were no products in pEGFP-C3/CHO cells, $\mathrm{CHO}$ cells, and the negative control. These results confirmed that stably transfected hCD300LG- $\gamma / \mathrm{CHO}$ cells could express hCD300LG- $\gamma$ mRNA. 


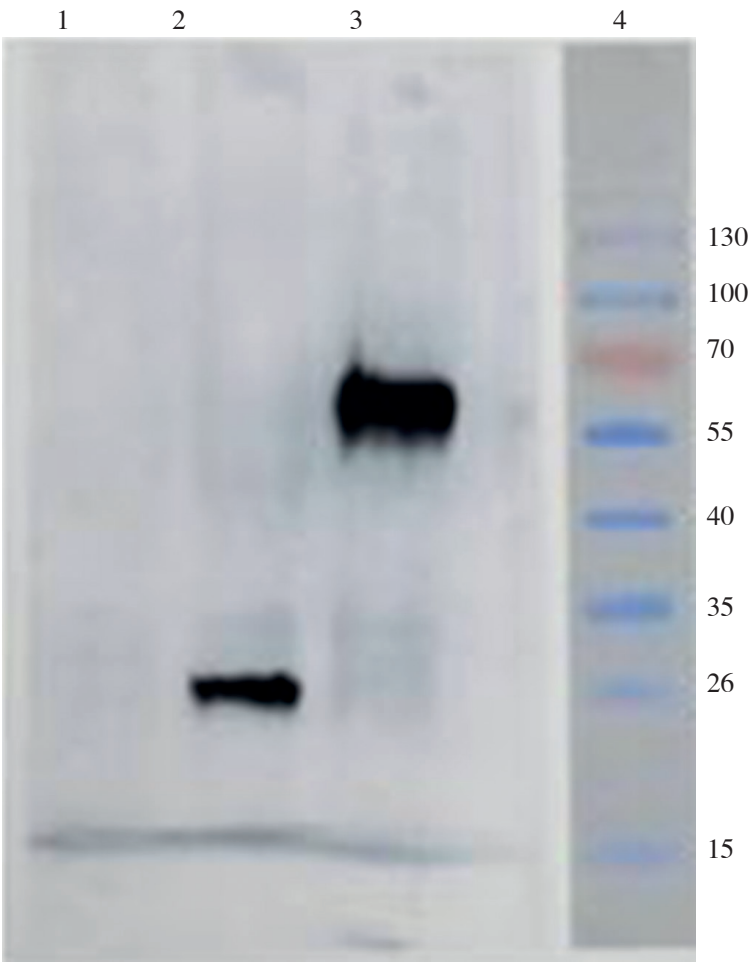

Fig. 3. Western blot analysis of protein expression: $1-$ whole lysates of $\mathrm{CHO}$ cells; 2 - whole lysates of pEGFP-C3/CHO cells; 3 - whole lysates of hCD300LG- $\gamma / \mathrm{CHO}$ cells; 4 - pre-stained protein marker

Western Blot: Western Blot results showed that anti-GFP antibodies could recognise cells lysates of pEGFP-C3/CHO and hCD300LG- $\gamma / \mathrm{CHO}$ cells but could not recognise $\mathrm{CHO}$ cell lysates (Fig. 3). Wherein, the molecular weight of the band of pEGFP-C3/CHO cell lysates was $27 \mathrm{KD}$ and the band of hCD300LG- $\gamma / \mathrm{CHO}$ cell lysates was about $60 \mathrm{KD}$, which was consistent with the expected size.

\section{The killing activities assay of induced CTL and NK cells}

On the basis of standard CIK inducing reagents addition, cell lysates of hCD300LG- $\gamma / \mathrm{CHO}$, pEGFP-C3/ $\mathrm{CHO}$, and $\mathrm{CHO}$ cells were, respectively, used to induce

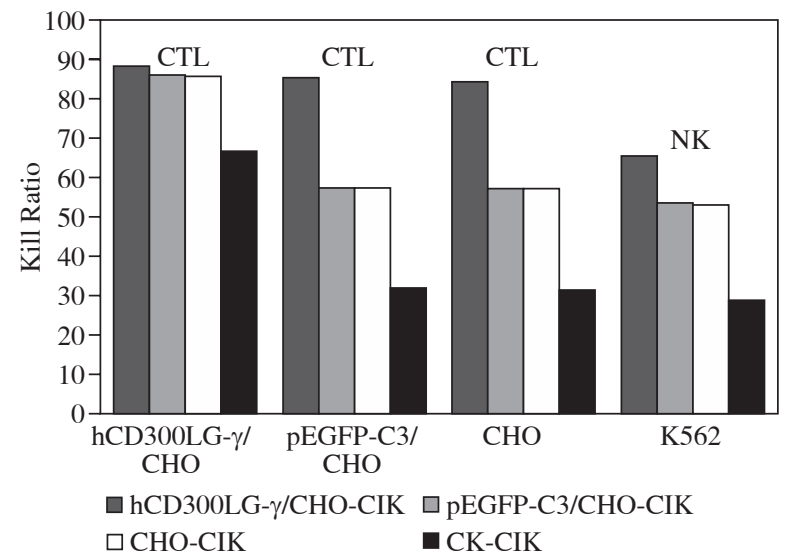

Fig. 4. The killing activities of induced CIK cells of CTL and NK

CIK and were recorded as hCD300LG- $\gamma / \mathrm{CHO}-\mathrm{CIK}$, pEGFP-C3/CHO-CIK, CHO-CIK, and CK-CIK. Using the four kinds of induced CIK cells as effector cells, and using hCD300LG- $\gamma / \mathrm{CHO}$, pEGFP-C3/CHO, CHO, and K562 as target cells, the killing activities of the four kinds of CIK cells to the four kinds of target cells were researched. The light absorption value (A value) was tested by AlamarBlue and is shown in Table 1. Then the killing ratio was calculated according to the formula: Killing ratio $=\left[1-\left(\mathrm{A}_{\text {test well }}\right.\right.$ $\left.\left.-\mathrm{A}_{\text {effector control well }}\right) / \mathrm{A}_{\text {target control wells }}\right] \times 100 \%$.

As shown in Fig. 4, the killing activities of CTL and NK cells of hCD300LG- $\gamma / \mathrm{CHO}-\mathrm{CIK}$ were clearly enhanced compared with the other three kinds of CIK, especially enhanced in killing pEGFP-C $3 / \mathrm{CHO}, \mathrm{CHO}$, and K562. The killing activities of pEGFP-C3/CHO-CIK and CHO-CIK were similar, which were elevated compared with CK-CIK and reduced compared with hCD300LG- $\gamma /$ CHO-CIK. The killing activities of CK-CIK were the lowest compared with the other three kinds of CIK, in particular they declined significantly compared with hCD300LG- $\gamma / \mathrm{CHO}-\mathrm{CIK}$.

\section{Discussion}

Adoptive cellular immunotherapy by infusion immunocompetent cells is not only a supplement to conventional

Table 1. Light absorption value tested by AlamarBlue method

\begin{tabular}{|c|c|c|c|c|c|}
\hline \multirow{2}{*}{$\begin{array}{l}\text { Effector cells } \\
\text { Target cells }\end{array}$} & CTL & ctl & CTL & NK & \multirow[b]{2}{*}{ Effector control } \\
\hline & hCD300LG- $\gamma /$ CHO-CIK & pEGFP-C3/CHO -CIK & CHO-CIK & CK-CIK & \\
\hline hCD300LG- $\gamma / \mathrm{CHO}$ & 0.256 & 0.282 & 0.284 & 0.419 & 0.189 \\
\hline pEGFP-C3/CHO & 0.274 & 0.473 & 0.472 & 0.661 & 0.188 \\
\hline $\mathrm{CHO}$ & 0.277 & 0.474 & 0.471 & 0.663 & 0.188 \\
\hline K562 & 0.364 & 0.477 & 0.479 & 0.660 & 0.167 \\
\hline Target control & 0.568 & 0.663 & 0.662 & 0.693 & \\
\hline
\end{tabular}


anti-tumour treatment, but also a new treatment approach for patients who are not suitable for surgery, radiotherapy, or chemotherapy [16]. Cytokine-induced killers (CIK) are a group of tumour-killing immunocompetent cells with high efficiency, broad-spectrum, and no MHC restriction [17-19]. At present, CIK used in adoptive immunotherapy is prepared by mononuclear cells separated from peripheral blood, bone marrow, or umbilical cord blood, which propagate after being stimulated by a variety of cytokines such as IL-1, IL-2, IFN- $\gamma$, and CD3McAb. Because CIK tumour killing has the characteristics of high efficiency, broad-spectrum, fewer side effects, and no MHC restriction, CIK displays great value for application in tumour immunotherapy [20]. The key in CIK therapy is to increase the cell number and killing activity, especially CTL and NK cells, which has powerful antitumor effects. Many researches aimed to enhance CIK killing activities through CIK combined vaccine, DC-CIK, cytokine gene transfection, combined molecular targeted drugs, and many other ongoing methods [18-20].

CD300LG can associate with L-selectin, which participates in lymphocyte homing. It mediates lymphocytes rolling, adhesion, and TEM dependent on L-selectin, and plays important roles in inflammation and immune surveillance $[2,3,5]$. Eiji Umemoto et al. found that CD300LG expression in microvascular endothelial cells was influenced by local factors produced in tissues. CD300LG expression was closely correlated with lymphocytes infiltration in certain tissues. For example, in tumour tissues, decreased expression of CD300LG might inhibit the activities of lymphocytes killing target cells, which might participate in tumour escape [13].

In conclusion, CD300LG, as an adhesion molecule, participates in lymphocyte adhesion, TEM, and migration to focus location. It might be able to improve the killing activities of antitumor cells, such as CTL and NK cells, to promote lymphocyte function. Therefore, in this study, CD300LG was used as an inducing factor to explore whether it enhanced the killing activity of CIK cells. The results might provide a basis for subsequent discussion on mechanisms.

Firstly, the eukaryotic expression plasmid hCD300LG- $\gamma /$ pEGFP-C3, which can express human CD300LG- $\gamma$, was constructed and transfected into $\mathrm{CHO}$ cells, which provided a basis for subsequent induction experiments. The effect of CD300LG induction on the killing activities of CIK cells was studied using cell lysates as an inducer. The results showed that the killing ratios of $\mathrm{pEG}$ FP-C3/CHO-CIK and CHO-CIK were similar; therefore, the impact of pEGFP-C3 plasmid on the result could be excluded. Also, the activities of killing CHO and K562 cells were similar between CHO-CIK and CK-CIK, so the impact of $\mathrm{CHO}$ cells on the result could be excluded. After hCD300LG- $\gamma$ induction, the activities of hCD300LG- $\gamma /$ CHO-CIK killing four types of target cells were all in- creased compared with the other three kinds of CIK. In particular, the activities of CTL killing pEGFP-C3/CHO and $\mathrm{CHO}$ cells were significantly enhanced. When using hCD300LG- $\gamma / \mathrm{CHO}$ as target cells, the killing activities had no significant difference in hCD300LG- $\gamma / \mathrm{CHO}-\mathrm{CIK}$, pEGFP-C3/CHO-CIK, and CHO-CIK, whereas they were all significantly higher than that of CK-CIK. The activities of CIK killing target cells of pEGFP-C3/CHO, $\mathrm{CHO}$, and $\mathrm{K} 562$ cells were similar, but they were significantly enhanced in killing hCD300LG- $\gamma / \mathrm{CHO}$ cells. Meanwhile the activities of CK-CIK killing hCD300LG- $\gamma / \mathrm{CHO}$ cells were significantly increased compared with that of killing pEGFP-C3/CHO, CHO, and K562 cells.

Our results indicated that after CD300LG induction, the activities of CIK including CTL and NK cells could be significantly enhanced. And compared with standard cytokine induction, CD300LG induction had a better effect. Therefore, CD300LG could be expected to become an important molecule in CIK induction to enhance antitumor effects. Interestingly, the activities of CIK killing hCD300LG- $\gamma / \mathrm{CHO}$ were higher than that of killing pEGFP-C3/CHO, CHO, and K562 cells, which could not express CD300LG- $\gamma$. This suggests that whether CD300LG was expressed on effector cells or on target cells, it might increase the number of local immunocompetent cells via mediating lymphocytes adhesion, thereby enhancing killing activities of CIK cells, such as CTL and NK cells. The exact mechanisms need to be further researched.

\section{The authors declare no conflict of interest.}

\section{References}

1. Umemoto E, Tanaka T, Kanda H, et al. (2006): Nepmucin, a novel HEV sialomucin, mediates L-selectin-dependent lymphocyte rolling and promotes lymphocyte adhesion under flow. J Exp Med 203: 1603-1614.

2. Takatsu H, Hase K, Ohmae M, et al. (2006): CD300 antigen like family member $\mathrm{G}$ : A novel $\mathrm{lg}$ receptor like protein exclusively expressed on capillary endothelium. Biochem Biophys Res Commun 348: 183-191.

3. Umemoto E, Takeda A, Jin S, et al. (2013): Dynamic changes in endothelial cell adhesion molecule nepmucin/CD300LG expression under physiological and pathological conditions. PLoS One 8: e83681.

4. Jin S, Umemoto E, Tanaka T, et al. (2008): Nepmucin/CLM9, an Ig domain-containing sialomucin in vascular endothelial cells, promotes lymphocyte transen-dothelial migration in vitro. FEBS Lett 582: 3018-3024.

5. Xiao-dong P, Zhi-juan L, Chong-jie Z (2010): Nepmucin, a novel adhesion molecule. Int J Immunol 33: 79-84.

6. Cavallaro U, Christofori G (2004): Cell adhesion and signalling by cadherins and Ig-CAMs in cancer. Nature Rev Cancer 4: 118-132.

7. Hazan RB, Qiao R, Keren R, et al. (2004): Cadherin switch in tumor progression. Ann N Y Acad Sci 1014: 155-163. 
8. Guo W, Giancotti FG (2004): Integrin signalling during tumor progression. Nat Rev Mol Cell Biol 5: 816-826.

9. Kim JB, Islam S, Kim YJ, et al. (2000): N-cadherin extracellular repeat mediates epithelial to mesenchymal transition and increased motility. J Cell Biol 151: 1193-1206.

10. Li LC, Chui RM, Sasaki M, et al. (2000): A single nucleotide polymorphism in the E-cadherin gene promoter alters transcriptional activities. Cancer Res 60: 873-876.

11. Mialhe A, Levacher G, Champelovier P, et al. (2000): Expression of E-, P-, N-cadherins and catenins in human bladder carcinoma cell lines. J Urol 164: 826-835.

12. Mueller S, Cadenas E, Schönthal AH (2000): p21 WAFL regulate sanchorage-independent growth of HCT116 coloncarcinoma cells via E cadherin expression. Cancer Res 60: 156-163.

13. Makrilia N, Kollias A, Manolopoulos L, et al. (2009): Cell adhesion molecules: role and clinical significance in cancer. Cancer Invest 27: 1023-1037.

14. Rychly J, Nebe B (2006): Therapeutic strategies in autoimmune diseases by interfering with leukocyte endothelium interaction. Curr Pharm Des 12: 3799-3806.

15. Zhai S, Xu H, Jiang X, et al. (2016): Expression Depression of CD300LG- $\gamma$ in Human Pulmonary Carcinoma. Monoclon Antib Immunodiagn Immunother 35: in printed.

16. DeVita VT Jr, Rosenberg SA (2012): Two hundred years of cancer research. N Engl J Med 366: 2207-2214.

17. Schmidt-Wolf IG, Negrin RS, Kiem HP, et al. (1991): Use of a SCID mouse/human lymphoma model to evaluate cytokine induced killercells with potent antitumor cell activity. J Exp Med 174: 139-149.

18. Hontscha C, Borck Y, Zhou H, et al. (2011): Clinical trials on CIK cells: first report of the international registry on CIK cells (IRCC). J Cancer Res Clin Oncol 137: 305-310.

19. Jakel CE, Schmidt-Wolf IG (2014): An update on new adoptive immunotherapy strategies for solid tumors with cytokine-induced killer cells. Expert Opin Biol Ther 14: 905-916.

20. Nie Xin, Liu Kan-Feng, Teng Shao-Xia (2007): The anti-tumor development of CIK and its clinical application. China Pract Med 2: 106-108. 